\title{
1 Title: Disentangling the aging network of a termite queen
}

\section{José Manuel Monroy Kuhn ${ }^{1,2, *}$, Karen Meusemann ${ }^{1,3}$ and Judith Korb ${ }^{1, *}$}

$4{ }^{1}$ Department of Evolutionary Biology \& Ecology, Institute of Biology I, Albert Ludwig University

5 of Freiburg, Hauptstr. 1, D-79104 Freiburg (i. Brsg.), Germany

$6{ }^{2}$ Computational Discovery Research, Institute for Diabetes and Obesity, Helmholtz Zentrum

7 München, Ingolstaedter Landstr. 1, D-85764 Neuherberg, Germany

$8{ }^{3}$ Australian National Insect Collection, CSIRO National Research Collections Australia, Clunies

9 Ross Street, Acton, ACT 2601, Canberra, Australia

10

11 * corresponding authors: judith.korb@biologie.uni-freiburg.de; manuel.kuhn@ helmholtz-

12 muenchen.de

\section{Abstract}

15 Background: Most insects are relatively short-lived, with a maximum lifespan of a few weeks, like

16 the aging model organism, the fruit-fly Drosophila melanogaster. By contrast, the queens of social

17 insects (termites, ants, some bees and wasps) can live for more than a decade. This makes social

18 insects promising new models in aging research providing insights into how a long reproductive life

19 can be achieved. Yet, aging studies on social insect reproductives are hampered by a lack of

20 quantitative data on age-dependent survival and time series analyses that cover the whole lifespan

21 of such long-lived individuals. We studied aging in queens of the drywood termite Cryptotermes

22 secundus by determining survival probabilities over more than 15 years and performed

23 transcriptome analyses for queens of known age that covered their whole lifespan. 
24 Results: The maximum lifespan of $C$. secundus queens was 13 years with a median maximum

25 longevity of 11.0 years. Time course and co-expression network analyses of gene expression

26 patterns over time indicated a non-gradual aging pattern. It was characterized by networks of genes

27 that became differentially expressed only late in life, namely after an age of 10 years, which

28 associates well with the median maximum lifespan for queens. These old-age gene networks reflect

29 processes of physiological upheaval. We detected strong signs of stress, decline, defence and repair

30 at the transcriptional level of epigenetic control as well as at the post-transcriptional level with

31 changes in transposable element activity and the proteostasis network. The latter depicts an

32 upregulation of protein degradation, together with protein synthesis and protein folding, processes

33 which are often down-regulated in old animals. The simultaneous upregulation of protein synthesis

34 and autophagy is indicative for a stress-response mediated by the transcription factor $c n c$, a

35 homolog of human $n r f$ genes.

36 Conclusion: Our results show non-linear senescence with a rather sudden physiological upheaval at

37 old-age. Most importantly, they point to a re-wiring in the proteostatis network as central for

38 explaining the long life of social insect queens.

39

\section{Keywords}

41 RNASeq, transcriptomes, ageing, social insects, weighted gene co-expression networks, WGCNA,

42 time series, termite, lifespan, senescence 


\section{Background}

44 Almost all animals age, but at different pace [1]. The fruit fly Drosophila melanogaster lives only

45 for around seven weeks [2], while the clam Ocean Quahog, Arctica islandica, can have a lifespan of

46 more than 400 years [3]. Generally, organisms with large differences in rates of aging are found

47 between widely divergent species $[1,4]$, which makes controlled comparisons of the underlying

48 aging mechanisms difficult.

49 Classical model organisms typically have a short lifespan and can be characterized by r-life history

50 strategies ('live fast, have many offspring and die young') as exactly these traits make them good

51 model organisms. Social insects such as termites, ants, or the honeybee, offer promising new

52 insights into aging research because individuals with the same genetic background can differ by

53 orders of magnitudes in lifespan. Within a social insect colony, which is generally a large family,

54 the reproducing queen (and in termites, also the king) can reach lifespans of more than 20 years,

55 while non-reproducing workers have a lifespan of a few months only [5-8]. However, quantitative

56 demographic data covering the whole lifespan of queens are inherently rare and many reports on

57 queen-longevity are more anecdotal. Thus, it is largely unknown for long-lived queens whether they

58 age gradually or whether aging is a more sudden event.

59 During recent years, several pioneering studies, especially on the honeybee, revealed exciting new

60 insights into the mechanisms of how queens can live so long. In the honeybee, juvenile hormone

$61(\mathrm{JH})$ seems to have lost its direct gonadotropic function in adults so that queens have a high

62 expression of the yolk precursor gene vitellogenin $(V g)$ without requiring high JH titres (e.g.,

$63[9,10])$. This result has led to the hypothesis that uncoupling JH and $V g$ expression might account

64 for the long life of honeybee queens [9] as well as social insect queens more generally [11] because

65 the life-time shortening consequences of high JH titers are absent. However, this re-wiring along the

$66 \mathrm{JH}-\mathrm{Vg}$ axis is not universal for all social Hymenoptera, since the queens of many other ant and bee 
67 species require $\mathrm{JH}$ for vitellogenesis (e.g. [12] and references therein). For termites, fewer studies

68 exist, but $\mathrm{JH}$ is required for vitellogenesis $[13,14]$. Hence, other mechanisms must exist to explain

69 the long life of termite queens. Studies of the subterranean termite Reticulitermes speratus

70 implicated the involvement of a breast cancer type 1 susceptibility (BRCA1) homolog [15], which is

71 involved in DNA repair [16], and better protection against oxidative stress by superoxide

72 dismutases and catalases $[17,18]$. The latter have also been discussed for other social Hymenoptera,

73 including ants and the honeybee. Yet overall evidence of the role of oxidative stress is less clear

74 (reviewed in [19-21]). Furthermore, regulation of the activity of transposable elements (TEs) [22],

75 and changes in the insulin/insulin-like growth factor1 signalling (IIS) and target of rapamycin

76 (TOR) pathways [23] have been linked with caste-specific aging differences in termites. Both, the

77 TOR and IIS pathway, have been associated with longevity in model organisms from $D$.

78 melanogaster to mice and humans [24-26]. They are the most intensively studied aging related

79 pathways and they have also been associated with caste differences in social Hymenoptera (e.g.,

80 [27-31]). Yet, all studies on social insects suffer from a lack of time-series data to investigate

81 molecular changes across the lifespan of long-lived queens. Like the demographic life history data,

82 such data are inherently difficult to obtain due to the long lifespan of queens. However, they are

83 necessary (i) to understand the aging process, (ii) to work out potential changes compared to

84 solitary insects, and (iii) to identify the relevant age-classes for detailed studies. The latter is a

85 completely overlooked issue but highly relevant. If, for example, aging is a non-linear process,

86 differences across studies might just be consequences of none-comparable age-classes between

87 studies.

88 We studied aging in termite queens of known age across their entire lifespan to measure at the

89 ultimate, eco-evolutionary level age-dependent survival and at the proximate, mechanistic level

90 age-specific changes in gene expression. For the latter, we generated head and thorax 
91 transcriptomes of queens of different age (for an outline of the workflow see Additional File 1,

92 Figure S1). We used field collected, newly established colonies of the wood-dwelling termite

93 Cryptotermes secundus (Hill, 1925) (Blattodea, Isoptera, Kalotermitidae) that were kept under

94 identical conditions in the laboratory for a time span of 15 years. Keeping them under constant and

95 optimal conditions allowed us to study intrinsic aging, disentangled from causes of extrinsic

96 mortality such as predation, food shortage, or disease. As typical for wood-dwelling termite species,

97 C. secundus colonies are founded in a piece of wood, which serves as food and shelter and which

98 workers never leave to forage outside. Such species have a low social complexity with small

99 colonies and totipotent workers that develop into sexuals.

100

\section{Results}

\section{Survival analysis}

103 Overall, we had surviving founding queens from an age of two years up to a maximum of 13 years.

104 The potentially 14- and 15-year old queens all had died as had most queens with a potential age of $\geq$

10512 years. Out of eight queens in this 'old-age' class, only a single queen (13 years) had survived

106 (Fig. 1). The median longevity of the queens in the laboratory after successful colony establishment

107 was estimated with Kaplan Meier survival analysis to be 12.0 years (SE: \pm 0.54 ) (mean longevity:

10811.1 years, SE: \pm 0.66$)($ Fig. 1$)$.

109

\section{Identifying transcripts that change their expression with age: age-related DETs}

111 To study gene expression changes over the lifetime of queens we generated transcriptomes of head

112 and thorax from twelve queens with different chronological age since onset of reproduction, from

113 two until 13 years, covering the complete lifespan of $C$. secundus queens: $2,3,4,5,6,7,8,9,10$

114 (two samples), 11, and 13 years (Additional File 2, Table S1). 
115 A total of 169 transcripts were significantly differentially expressed (DETs) over time as revealed

116 by Iso-MaSigPro time series analysis (Additional File 2, Table S2). According to their expression

117 pattern, DETs were grouped into six Iso-MaSigPro clusters (hereafter, 'cluster') (Fig. 2). Cluster 1

118 represented 44 DETs, which were slightly expressed in young queens followed by a decline at

119 middle ages and a strong increase when queens became older. The 32 DETs of cluster 2

120 characterized young queens with a declining expression with age. Clusters 3 and 5 comprised 31

121 and 37 DETs, respectively, that were highly expressed in middle aged queens, while cluster 4 and

122 cluster 6 (15 and 10 DETs) characterized old queens with no expression in young ones. Thus, in the

123 following we referred to the DETs as young (cluster two), middle-aged (clusters three and five) and

124 old DETs (clusters one, four and six). Details for all clusters are provided in Additional File 2,

125 Table S2.

126

127 Identifying modules of co-expressed transcripts

128 To identify modules of co-expressed transcripts, we performed a weighted gene co-expression

129 network analysis (WGCNA). It revealed a total of 254 modules of co-expressed transcripts. Based

130 on eigengene values, 13 modules correlated significantly positively with age and 13 negatively (see

131 Additional File 1, Figures S2 and S3; Additional File 3 (WGCNA module-age association, shown

132 are eigengene values for all modules).

133

\section{Identifying transcript co-expression modules with age-related DETs}

135 Within the age-correlated WGCNA modules, we identified age-related DETs. The negatively age-

136 correlated module 'seashell4' had the highest number of young DETs (10 DETs). No gene ontology

137 (GO) term was enriched for this module. The highest number of old DETs were found in the

138 positively age-correlated modules ‘cyan' (89 DETs) and ‘tan’ (79 DETs) (Additional File 2, Table 
139 S3 and S4). Only broad categories were enriched in the 'cyan' module (e.g. RNA metabolic process

140 and gene expression) and the 'tan' module was enriched for ribosomal and tRNA related functions

141 (Additional File 1, Figure S4).

142

\section{Extracting age-related subnetworks based on age-related DETs}

144 To generate subnetworks related to the age-related DETs, we located them in the WGCNA co-

145 expression network. These DETs and their one- and two-step neighbors (i.e., the 'second level

146 neighborhood') were then extracted from the co-expression network, which resulted in 50

147 subnetworks of different sizes (for more details see Methods) (Additional File 1, Figure S5). Note,

148 DETs might be located at the boundaries of multiple WGCNA modules which means the

149 subnetworks obtained consist of fragments of multiple WGCNA modules. The resulting

150 subnetworks either contained young and middle-aged DETs or old DETs, with a single exception

151 where a middle-aged DET was in the periphery of the largest subnetwork containing old DETs. The

152 largest subnetwork containing either young and middle-aged DETs (hereafter, young subnetwork)

153 or old DETs (plus a single middle-age DET; hereafter old subnetwork) were further analyzed.

\section{4}

\section{Young subnetwork}

156 The largest young subnetwork comprised 164 transcripts (out of these 24 Iso-MaSigPro DETs) of

157 which only 12 (7\%) were one-to-one orthologs to D. melanogaster genes (Additional File 2, Table

158 S3). The GO enrichment analysis of the young subnetwork showed multiple Biological Process

159 (BP) terms related to RNA catabolism, but these GO terms were not significant after correcting for

160 multiple testing (Additional File 2, Figure S6).

161 
$162 T E$ activity and genome instability. 53 DETs (32\%) of the young subnetwork were related to TEs

163 (Fig. 3 and Additional File 2, Table S3), comprising TEs and genes from TE defense pathways.

164 This included one homolog of the gene argonaute 2 (ago2) (two transcripts), an essential gene of

165 the endo-siRNA pathway which silences TEs [32], and arsenite 2 (ars-2) which is required for

166 siRNA and miRNA-mediated TE silencing [33]. Additionally, we found two genes connected to

167 DNA damage response and genome instability: kin17 and PIF1-like gene.

169 Other signatures. From well-known aging pathways, we identified (i) inositol polyphosphate

170 phosphatase 2 (mipp2) and (ii) adenylyl cyclase $76 E$ (ac76E). The former is part of the TOR

171 pathway and has been associated with longevity [34] and the latter is activated by the transcription

172 factor 'Forkhead box O' (foxo). Additionally, we found several fecundity related DETs. They

173 included two transcripts of the gene hu li tai shao (hts) (one a DET of IsoMaSigPro cluster two) and

174 one homolog of the gene bällchen (ball) (two transcripts) (one a DET of cluster five, Fig. 3)

\section{Old subnetwork}

177 The largest 'old subnetwork' comprised 1,098 transcripts (out of these 42 Iso-MaSigPro DETs).

178521 transcripts (47\%) were identified as one-to-one orthologs of D. melanogaster genes (Additional

179 File 2, Table S4). Iso-MaSigPro DETs in the old subnetwork belonged mainly to Iso-MaSigPro

180 clusters 1 and 4 . The second level neighborhoods of these DETs were connected in the network and

181 a GO enrichment analysis revealed multiple GO terms associated with protein related functions,

182 including translation, protein folding, unfolded protein binding, proteolysis involved in cellular

183 protein catabolic process, protein targeting to ER, ribosome, and proteasome complex (Additional

184 File 1, Figures S7 and S8). 198 transcripts of the old subnetwork (18\%) were involved in protein

185 translation, protein folding, and protein catabolism and proteolysis (Figs. 4 and 5 [35,36] and 
186 Additional File 2, Table S4). Additionally, 61 transcripts ( $6 \%)$ were related to TEs (Additional

187 File 2, Table S4).

189 Epigenetic modifications, transcriptional regulation, and TE activity. Many genes of the old

190 subnetwork are involved in de/acetylation and methylation of DNA, which are important epigenetic

191 modifications that regulate gene expression and genome stability [37-39] (Additional File 2, Table

192 S4).

193 Most strikingly, two crucial histone acetylation modifying complexes, the Tip60 acetyltransferase

194 complex and the male specific lethal (msl) complex were represented in the old subnetwork. The

195 former included the genes dom, ing3, mrg 15, pont and rept, and the latter msl-1, msl-3 and mof.

196 Genes involved in deacetylation of DNA were, for instance, sirtuin 1 (sirt1), histone deacetylase 3

197 (HDAC3), and histone deacetylase 6 (HDAC6). Genes linked to epigenetic histone methylation

198 included, for instance, ash-1 and lid. Another well represented group of genes connected to

199 expression regulation in the old subnetwork were spliceosome components and splicing factors.

200 Additionally, we found in the old subnetwork important transcripts related to TE silencing: dicer-2,

201 Hsc70-4, Hsc70-3, Hsp83, trsn, armi, Rm62, Gasz, Tudor-SN, and Hel25E. Details are given in

202 Additional File 2, Table S4.

203

204 Proteostasis and oxidative stress. Related to proteostasis we detected a strong signal for protein

205 synthesis and degradation. Regarding protein synthesis, the old subnetwork comprised many

206 transcripts coding for initiation, elongation and termination factors, as well as many ribosomal

207 proteins and aminoacyl-tRNA synthetases (Fig. 4; Additional File 2, Table S4). Regarding protein

208 degradation, almost all subunits of the ubiquitin proteasome system (UPS) were present (Fig. 5) as

209 well as autophagy genes, heat shock proteins, and the transcription factor $x b p 1 . X b p 1$ is involved in 
210 the 'unfolded protein response' (UPR) and in the ER-associated protein degradation (ERAD)

211 pathway $[40,41]$.

212 Additionally, BRCAl was also present in the old subnetwork. This gene is involved in oxidative

213 stress response, and in the transcriptional activation of proteasomal genes by stabilizing the

214 transcription factor cnc/nrf-2 (cap-n-collar/nuclear factor erythroid 2-related factor 2) [42]. Other

215 genes in the old subnetwork involved in oxidative stress response and transcriptionally activated by

$216 n r f-2$ were thioredoxin and S-glutathione transferase.

217

218 Other signatures. Additionally, the old subnetwork was characterized by a signature of ecdysone

219 biosynthesis with ecdysone receptor (EcR), ecdysone-induced protein 75B (Eip75B), phantom and

220 disembodied. The presence of Phosphotidylinositol 3 kinase 68D (Pi3K92E) links to the IIS

221 pathway.

222

\section{Locating age-related co-expression modules in the age-related subnetworks}

224 Finally, we inspected those WGCNA modules with a large fraction of transcripts in the young and

225 old subnetworks as well as those modules that were significantly associated with age.

226 In the young subnetwork, WGCNA modules with a large fraction of transcripts were 'saddlebrown'

227 and 'skyblue4' which both did not significantly correlate with age. Significantly age-correlated co-

228 expression modules were firebrick2, indianred1 and seashell4 (Additional File 1, Figure S2). No

229 GO terms were significantly enriched for any of these modules.

230

231 In the old subnetwork, the modules with a large fraction of transcripts were 'green' and

232 'paleturquoise' which again did no significantly correlate with age. The old subnetwork contained

233 transcripts of 13 significantly age-correlated co-expression modules (Additional File 1, Figure S3). 
234 The GO enrichment analysis of these modules revealed several terms involved in protein related

235 functions, including ribosome biogenesis, rRNA processing, protein folding, translation, unfolded

236 protein binding, protein catabolic process, protein transport, tRNA aminoacylation for protein

237 translation, and proteasome core complex (Additional File 1, Figure S4, S9, S10 and Additional

238 File 4, Table S5).

\section{Discussion}

241 Our study revealed a median maximum reproductive longevity of $C$. secundus queens of 12 years

242 with a maximum lifespan of 13 years when excluding all causes of extrinsic mortality in the

243 laboratory. The curve suggests a rather sudden decline in live expectancy after an age of around 11

244 years; out of eight queens with a potential age $\geq 12$ years, all had died, except one 13-year old

245 queen (Fig. 1). The survival curve indicates a type I survivorship, after queens have successfully

246 founded a colony and without extrinsic mortality.

248 Our transcriptome study identified six clusters of transcripts that were significantly differentially

249 expressed with age (DETs) (Fig. 2): one cluster for young queens (cluster 2), two for medium aged

250 queens (cluster 3 and 5) and three for old queens (cluster 1, 4, and 6). This implies that three

251 'molecular' life stages can be distinguished in C. secundus queens with the third corresponding to

252 old, aged queens that will probably die soon as no queen reached a lifespan beyond 13 years. The

253 co-expression network analysis, which extracted subnetworks based on age-related DETs, resulted

254 in two main subnetworks, a young and an old subnetwork. This implies that there are two age-

255 related 'molecular' life stages, as DETs/genes of young and medium ages belonged to the same

256 young subnetwork. 


\section{Young subnetwork}

259 The young subnetwork contained DETs characteristic for young and medium ages. This shows the

260 similarity in expression of these two age stages. Not unexpectedly, the young subnetwork indicates

261 an upregulation of transcripts linked with fecundity (e.g., hts, ball) and of the TOR pathway

262 (mipp2) which has been associated with longevity [34]. The upregulation of Ac76E may imply that

263 the IIS pathway is down because this gene is activated by the transcription factor foxo which is

264 inhibited by an upregulated IIS pathway. However, other evidence suggests that, like in other social

265 insects (e.g. [12] and references therein), queens are characterized by an upregulated IIS pathway

266 [23]. Additionally, we detected several upregulated TEs-related transcripts associated with signs of

267 an upregulation of the endo-siRNA pathway (e.g. ago2, ars) which is a transcriptional and post-

268 transcriptional TE-defence mechanism of the soma [32,33,43] (Kim, Lee and Carthew, 2007; Sabin

269 et al., 2009; Piatek and Werner, 2014).

\section{Old subnetwork}

272 The old subnetwork contained many more transcripts (1,098 vs. 164 in the young subnetwork). Our

273 results imply a physiological stage of upheaval shortly before queens die. There were strong signs

274 of decline and repair at the upstream transcriptional level of epigenetic control as well as at the

275 posttranscriptional level of TE-activity and the proteostasis network.

\section{Epigenetic modifications}

278 An upregulation of genes modifying histone marks implied considerable epigenetic changes that

279 lead to altered gene expression as is typical for aging organisms:

280 First, our results indicate dynamic changes of 'active' histone marks of euchromatin because many

281 genes related to $\mathrm{H} 3 \mathrm{~K} 4$ and $\mathrm{H} 3 \mathrm{~K} 36$ de/methylation and $\mathrm{H} 4 \mathrm{~K} 16$ de/acetylation were present in the 
282 old subnetwork (Additional File 2, Table S4). For instance, the Tip60- as well as the msl-complex

283 were well represented. Both complexes are involved in acetylation of histones, including H4K16,

284 which, for instance, is indicative for replicative aging in yeast [44]. The upregulation of Sirt1 may

285 function as an antagonist as it deacetylates H4K16. The same applies for the deacetylases HDAC6

286 and HDAC3, which can also deacetylase histones [39].

287 Second, there is also evidence for changes of repressive histone mark of heterochromatin (e.g.

288 linked to H3K9 and H3K27 acetylation) (Additional File 2, Table S4). For instance, the old age

289 transcript ALP1 is an antagonist of HP1, the latter is involved in the maintenance of

290 heterochromatin. HP1 generally decreases with age, and its overexpression can lead to an increased

291 lifespan in D. melanogaster [45,46].

292

293 TE activity

294 In line with a deregulation of repressive histone mark of heterochromatin, several TE-related

295 transcripts were also connected with the old subnetwork (Additional File 2, Table S4). TEs often

296 are accumulated in heterochromatin which silences their activity [47]. Yet, dysregulation of

297 heterochromatin can allow them to become active and this has been associated with aging $[48,49]$.

298 Also the upregulation of several genes from two TE-defense pathways - the endo-siRNA pathway

299 (e.g. dicer2, Hsc-70-4, trsn) as well as the of piRNA pathways (e.g., piRNA biosynthesis. armi,

300 gasz, Hel25E, Rm62; ping-pong cycle: Tudor-SN, qin) - support the notion of active TEs. Both

301 pathways silence TEs posttranscriptionally [50-52]. TE activity and especially the piRNA pathway

302 has also been associated with aging and the longevity of termite reproductives in another termite

303 species [22].

304

305 Loss of proteostasis 
306 Our results revealed a very strong proteostasis signal indicative for an upheaval in protein

307 synthesis, protein folding and protein degradation in old queens. Many genes from the proteostasis

308 network were detected in the old subnetwork (Additional File 2, Table S4). They indicate an

309 upregulation of protein degradation, together with protein synthesis and protein folding (Figs. 4 and

310 5). This is unusual because old organisms are typically characterized by a downregulation of all

311 these processes (Fig. 6).

312

313 Protein synthesis. Many transcripts coding for ribosomal proteins and aminoacyl-tRNA synthetases

314 were found in the old subnetwork, indicative of upregulated protein synthesis (Additional File 2,

315 Table S4). This is further supported by a strong signal of an active TORC1 system which promotes

316 protein synthesis (Fig. 7 [53-55]). Thus, for instance, many downstream eukaryotic initiation

317 factors (eIF4A, eIF4B, eIF4E, eIF4G) and eukaryotic elongation factor (eEF2) transcripts were

318 found in the old subnetwork (Fig. 4). They activate, ribosome biogenesis, translational elongation,

319 and cap-dependent translocation (Fig. 7).

320

321 Protein degradation. Normally, an active TORC1 system is associated with a downregulation of

322 protein degradation as it inhibits proteolytic systems [54-56] and autophagy (e.g., upregulated

323 TORC1 inhibits ATG1, which is necessary for autophagy activation; Fig. 7). Surprisingly,

324 however, we found strong evidence of upregulated protein degradation in the old subnetwork.

325 Several transcripts linked to autophagy, almost all subunits of the UPS, the UPR-, and the ERAD

326 pathway as well as heat shock proteins characterized the old subnetwork (Figs. 5 and 6; Additional

327 File 2, Table S4).

328 
329 Linking protein synthesis and degradation. The simultaneous upregulation of protein synthesis and

330 autophagy might be explained by a stress response. In D. melanogaster as well as in humans, under

331 stress conditions upregulated TORC1 enhances an oxidative stress response, controlled by the

332 transcription factor $c n c$, a homolog of human $n r f$ genes (Fig. 7). Ubiquitinated proteins and

333 damaged mitochondria activate $c n c / n r f-2$ via p62, supported by upregulated TORC1, which then

334 activates oxidative stress response genes [57,58] (Ichimura et al., 2013; Aramburu et al., 2014).

335 Additionally, $c n c$ is known to activate chaperones (protein folding) and the proteasome [59], and

336 this has been associated with lifespan expansion in D. melanogaster and Caenorhabdtis elegans

$337[60,61]$.

338 Support for the conclusion of a stress-associated, active $c n c$ transcription factor in old queens

339 comes from several transcripts in the old subnetwork (Fig. 7): (i) BRCA1, which indirectly actives

$340 c n c$ by inhibiting $c n c$ inhibitor Keapl, and (ii) the Tip 60 complex as well as genes that are

341 transcriptionally activated by cnc, such as thioredoxin, S-Glutathione transferases and UPS genes

342 (Fig. 7).

343 Hence, our results imply that old $C$. secundus queens are in a stage of stress. They have mounted

344 stress response systems mediated by $c n c$, including protein degradation and protein folding.

345 However, it is unusual that old queens can do this. In D. melanogaster, only young individuals can

346 mount this stress response [59] (Tsakiri et al., 2013). The constant activation of the proteasome in

347 these very old queens may lead to their death (note, the studied queens had reached their maximum

348 lifespan, we never had older queens) as the constant activation of the proteasome in transgenic flies

349 was detrimental for survival [59].

350

351 Comparison with other social insects 
352 There has been a debate about the role of oxidative stress to explain the long lifespan of social

353 Hymenoptera queens; yet evidence is inconclusive (e.g. reviewed by [19-21]). For instance,

354 markers of oxidative stress in the brain of honey bee workers do not increase with age, although

355 they live shorter than queens [62]. In the ant Lasius niger, both workers and males show higher

356 activity of the antioxidant superoxide dismutase than queens, but both live shorter than queens

357 [63]. These studies have shown that higher expression of oxidative stress response genes do not

358 necessarily correlate with longer lifespans. For the termite Reticulitermes speratus, it has been

359 suggested that queens are better protected against oxidative stress as qRT-PCRs studies showed a

360 higher expression of the antioxidants catalases and peroxiredoxins in queens compared to workers

361 [17], while kings were characterized by a high expression of BRCAl (in the fat body) compared to

362 workers [15]. Unfortunately, the age of the studied reproductives is not known. It would be of

363 interest to study expression of these genes with age, as this would contribute to a better

364 understanding of the aging process but such studies are rare.

\section{Conclusion}

369 Our results imply that $C$. secundus queens do not age gradually, rather at old age there is a

370 physiological stage of upheaval, characterized by signs of stress (activity of TEs, active $\operatorname{crc}$ ) and

371 defense (piRNA pathways) / repair (protein degradation and synthesis) before the animals die.

372 This apparently sudden decline is in line with the few life history records of social insect queens

373 that exist. They also found no signs of gradual senescence but an abrupt death (e.g., the ant

374 Cardiocondyla obscurior [64]; see also Fig. 1). This stresses the importance of using queens of

375 known age for aging studies as processes revealed from middle-aged versus old queens probably 
bioRxiv preprint doi: https://doi.org/10.1101/2020.12.19.423576; this version posted December 20, 2020. The copyright holder for this preprint (which was not certified by peer review) is the author/funder. All rights reserved. No reuse allowed without permission.

376 differ considerably. Our study is the first addressing the aging process for a social insect by

377 studying the complete lifespan of queens. 


\section{Methods}

379 Figure S1 (Additional File 1) provides a schematized workflow of the analyses described in the

380 following sections.

\section{Collection and colony maintenance}

383 From 2002 until 2016, C. secundus colonies were collected from mangroves near Palmerston -

384 Channel Island (12³0’ S, $131^{\circ} 00^{\prime}$ E; Northern Territory, Australia) when they were less than one

385 year old [65]. Colonies of an age of less than one year can be identified by the size and slightly

386 lighter sclerotization of the founding reproductives (primary reproductives), the presence of less

387 than 20 workers and short tunnel systems of a few centimeters. All collected colonies were

388 transferred to Pinus radiata wood blocks and transported to the laboratory in Germany, where they

389 were kept under standardized conditions in a climate room with a temperature of $28{ }^{\circ} \mathrm{C}, 70 \%$

390 humidity and a 12h day/night rhythm (Additional File 2, Table S1). Under these conditions,

391 colonies develop like in the field (see [66]). Up until 2019 wood blocks were split and the colonies

392 were extracted to determine survival of reproductives.

\section{Survival analysis}

395 The survival of primary queens (and kings) was determined by their presence /absence. Founding

396 (primary) queens can be identified by their dark brown color, compound eyes and wing abscission

397 scars. If the primary reproductives had died and the colony was still alive, they had been replaced

398 by neotenic replacement reproductives which lack these traits. The median longevity of queens was

399 determined using Kaplan Meier survival analysis in SPSS 23 [67]. Only colonies that survived the

400 transfer from the field to the laboratory in Germany and the re-establishment in the new wood block

401 were used for this analysis. This resulted in a sample size of 41 colonies. Overall, we had surviving 
402 primary queens from an age of one year up to a maximum of 13 years. The three potentially 14 - and

403 15-year old queens were all dead.

404

405 Transcriptome study

406 RNA extraction and sequencing

407 RNA was extracted from twelve queens with different chronological age since onset of

408 reproduction from two years until 13 years: 2, 3, 4, 5, 6, 7, 8, 9, 10 (two samples), 11, and 13 years.

409 In colonies older than 13 years, the original queen was always replaced by a neotenic replacement

410 queen.

411 An in-house protocol was followed for RNA extraction (see [23]). Individuals were placed on ice

412 and the gut was removed and discarded. The head together with the thorax were used for RNA

413 extraction. Samples were transferred into peqGOLD Tri Fast ${ }^{\mathrm{TM}}$ (PEQLAB) and homogenized in a

414 Tissue Lyser II (QIAGEN). Chloroform was used for protein precipitation. From the aqueous phase,

415 RNA was precipitated using Ambion isopropyl alcohol and then washed with $75 \%$ ethanol.

416 Obtained pellets were solved in nuclease-free water. DNA was subsequently digested using the

417 DNase I Amplification Grade kit (Sigma Aldrich, Cat. No. AMPD1). We performed an RNA

418 Integrity Number Analysis (RIN Analysis) measuring the RNA concentration with the Agilent

419 RNA 6000 Nano Kit using an Agilent 2100 Bioanalyzer (Agilent Technologies) for quality control.

420 Samples with total RNA were sent on dry ice to Beijing Genomics Institute (BGI) Tech Solutions

421 (HONGKONG) Co. and then to the BGI-Shenzhen (PR China) for sequencing. The preparation of

422 the cDNA libraries was performed by BGI according to their internal and proprietary standard

423 operating procedure. The cDNA libraries were paired-end sequenced (not-strand specific) on

424 Illumina HiSeq 2500 and 4000 platforms (100 base pairs read length and about 4 Giga bases per

425 sample). Index sequences from the machine reads were demultiplexed and a quality -check and 
426 filtering of raw reads was done using the package soapuke (-n 0.05 -1 20 -q 0.2 -p 1 -i -Q 2 -G --

427 seqType 1 and -A 0.5, http://soap.genomics.org.cn/).

429 Processing of RNASeq raw reads

430 FastQC (v0.11.4) [68] was used to evaluate the quality of the cleaned raw reads. To obtain a

431 transcript count table, the cleaned raw reads were pseudo-aligned with Kallisto (default settings,

432 v0.43.0) [69] (Bray et al., 2016) against a C. secundus transcriptome obtained from a draft version

433 of the C. secundus genome (with estimated gene and transcript models, see $[23,70]$. The counts

434 estimated by Kallisto were normalized using DESeq2 (v1.18.1, count/size factor) [71] in R (v3.4.4)

435 [72].

436

437 Time course analysis to identify age-associated differentially expressed transcripts (DETs)

438 The normalized counts were used as input for the R package Iso-MaSigPro (v1.50.0) [73] to test for

439 differentially expressed transcripts (DETs) across time. Iso-MaSigPro is designed for the analysis of

440 multiple time course transcriptome data. It implements negative binomial generalized linear models

441 (GLMs) [73,74]. Significantly differentially expressed transcripts (FDR corrected p-value set to

4420.05 ) were clustered using the clustering algorithm mclust in Iso-MaSigPro [73] resulting into six

443 Iso-MaSigPro clusters (Additional File 2, Table S2).

444

445 Weighted Gene Co-expression Network Analysis (WGCNA) to identify networks of co-expressed

446 transcripts

447 To obtain networks of co-expressed transcripts that were categorized as modules we performed a

448 Weighted Gene Co-expression Network Analysis (WGCNA). The counts obtained with Kallisto

449 (v0.43.0) [69] were transformed using variance stabilizing transformation (vst) as implemented in 
450 DESeq2 (v1.18.1) [71]. The vst transformed counts were used to perform a co-expression network

451 analysis with the R package WGCNA (v1.63) [75]. For more details on the methodology, see [75-

452 77]. In short, (Additional File 1, Figure S1, workflow, right side), a similarity matrix was built by

453 calculating Pearson correlations between the expression values of all pairs of transcripts. Using the

454 similarity matrix, a signed weighted adjacency matrix was obtained as described by the formula:

$$
a_{\mathrm{ij}}=\left(\frac{1}{2}\left(1+\operatorname{cor}_{\mathrm{ij}}\right)\right)^{\beta}
$$

455 Where cor $_{\mathrm{ij}}$ is the Pearson correlation between the expression pattern of transcript ' $i$ ' and transcript

456 ' $\mathrm{j}$ ' (the similarity value). The value of $\beta$ was chosen based on the soft-thresholding approach [75].

457 With this value of $\beta$, we obtained a weighted network with an approximate scale-free topology

$458\left(\beta=14\right.$, scale-free topology $\left.R^{2}=0.84\right)$. In a signed weighted adjacency matrix negative and small

459 positive correlations get negligibly small adjacency values shifting the focus on strong positive

460 correlations. Seeing the adjacency matrix as a network, the nodes correspond to the transcripts and

461 the connections between nodes correspond to the adjacency values (transformed correlation

462 coefficients). A topological overlap matrix (TOM), which in addition to the adjacency matrix

463 considers topological similarity (shared neighbors reinforce the connection strength between two

464 nodes), was constructed using the adjacency matrix [78]. To define transcript modules, a

465 hierarchical clustering tree was constructed using the dissimilarity measure (1-TOM). Transcript

466 modules were defined by cutting the branches of the tree using the Dynamic Hybrid Tree Cut

467 algorithm [79] and the minimum module size was set to 30 transcripts. Transcript modules with

468 similar expression profile were merged by hierarchical clustering of the eigengene correlation

469 values. Briefly, a hierarchical clustering tree was created with the eigengene dissimilarity measure

470 (1-correlation coefficient of eigengenes) and a tree height cut of 0.20 was used (corresponds to a

471 eigengene cor $\geq 0.80$ ). Eigengenes were calculated with the function moduleEigengenes (default

472 settings) [75]. A module eigengene corresponds to the first principal component of the module and 
473 can be seen as a weighted average expression profile [75]. To find significantly associated modules

474 with age, correlations between age and eigengenes of the merged modules were calculated. Each

475 module was named after a color by WGCNA.

476 The adjacency matrix of the WGCNA was visualized using Cytoscape (v3.7.1) [80], only including

477 pairs of nodes with cor $_{\mathrm{ij}} \geq 0.90$. The color of each module corresponds with the respective module

478 name (e.g., saddlebrown color for the saddlebrown module).

479

480 To identify co-expression modules containing age-related DETs, we looked for age-related DETs

481 from the Iso-MaSigPro analysis in the WGCNA modules. Those modules which were significantly

482 correlated with age and which contained the highest number of Iso-MaSigPro DETs were further

483 inspected.

484

485 Identifying/Extracting age-related subnetworks based on age-related DETs

486 To identify age-related subnetworks within the co-expression network, we combined the results of

487 the Iso-MaSigPro analysis with those from the WGCNA and extracted subnetworks that were based

488 on age-related Iso-MaSigPro DETs. Therefore, we extracted $1^{\text {st }}$ and $2^{\text {nd }}$ neighbors of DETs based on

489 the WGCNA co-expression network (i.e., the visual representation of the adjacency matrix). To do

490 this, we used the 'First neighbors' function of Cytoscape. We selected an age-related DET from Iso-

491 MaSigPro as transcript of interest. By calling the function, the neighboring transcripts were

492 selected, which were then extracted to form a subnetwork. By calling the function twice, one

493 obtains the one- and two-step neighbors (called 'second level neighborhood') of the transcript of

494 interest. This was done for each DET identified in IsoMaSigPro.

495 
496 The obtained subnetworks were clearly separated in those containing young Iso-MaSigPro DETs

497 (young subnetworks) and those containing old Iso-MaSigPro DETs (old subnetworks). The largest

498 subnetwork obtained for each group was used for further analysis paying attention to both,

499 transcript identity as well as WGCNA module content. Thus, we looked, for instance, for WGCNA

500 modules that had been identified to be age-related within the global WGCNA in these subnetworks.

501 The AutoAnnotate Cytoscape plug-in (v1.3) [81] was used to annotate the subnetworks using the

502 clustering algorithm 'Markov Cluster' (MCL) [82] to define and visualize sub-clusters, and the

503 labeling algorithm 'Adjacent Words' to label the sub-clusters. The Cytoscape plug-in BiNGO

504 (v3.0.3) [83] was used for GO enrichment analysis. The p-values of the GO enrichment analysis

505 were adjusted for multiple testing using the FDR approach [84]. Subnetworks were graphically

506 processed with Inkscape (v0.91, www.inkscape.org).

507

508 Transcript (functional) annotation

509 Nucleotide and protein sequences were obtained from the draft version of $C$. secundus genome

$510[23,70]$. For annotation, the translated transcripts were searched against the Pfam database (Pfam A,

511 release 30) [85] with the software hmmscan (option --cut_ga, HMMer v.3.1b2) [86] and against the

512 InterPro database with the software InterProScan (v5.17-56.0) [87]. Additionally, we did a

513 BLASTX search (NCBI BLAST suite v. 2.3.0) [88] with an e-value of $1 \mathrm{e}^{-05}$ as cut-off against the

514 protein coding sequences of the termite Zootermopsis nevadensis (official gene set version 2.2)

515 [89]. To further assist the annotation, we inferred a set of clusters of orthologous sequence groups

516 (COGs) from the official gene sets at the amino acid level of $C$. secundus (draft version) and $D$.

517 melanogaster, and a BLASTP search of $C$. secundus sequences against the protein coding

518 sequences (longest isoforms only) of D. melanogaster with a threshold e-value of $1 \mathrm{e}^{-05}$. 
519 To detect possible TEs, transcripts were searched against the Dfam database (v2.0) [90] using 520 nhmmer [91]. A transcript was considered TE related if there was a hit against the Dfam database

521 and the other annotation sources (Pfam, Interpro and BLAST) were not pointing in the direction of

522 a known gene.

\section{Gene identification and construction of gene trees}

525 In addition to the functional annotation, we inferred phylogenetic trees for selected transcripts

526 (Supplementary Archive 1, DRYAD, doi available upon acceptance of the manuscript). Following

527 the procedure described in [23], we retrieved protein coding sequences of the respective cluster of

528 orthologous sequence groups (COGs) from OrthoDB v.9.1 [92] for the following species: $D$.

529 melanogaster (DMEL), Apis mellifera (AMEL), Cardiocondyla obscurior (COBS), Polistes

530 canadensis (PCAN), Tribolium castaneum (TCAS), Z. nevadensis (ZNEV) and Blattella germanica

531 (BGER). COGs were identified using text search by searching for the gene name or IDs of $D$.

532 melanogaster. In case Selenocysteine (U) was included in sequences, "U” was replaced by " $\mathrm{X}$ “ to

533 avoid problems in downstream analyses since many programs cannot handle Selenocysteine

534 properly. Protein sequences of COGs of above listed species were separately aligned with MAFFT

535 (v.7.294b) applying the G-INS-i algorithm and otherwise default options [93]. For each multiple

536 sequence alignment, a profile hidden Markov model (pHMM) was built with hmmbuild (HMMER

537 v.3.1b2) [86]. Then the pHMM was used to search (hmmsearch) for corresponding protein coding

538 sequences in C. secundus and Macrotermes natalensis to identify orthologous candidate sequences

539 for each COG in both species. For gene (transcript) tree inference, we only kept sequences with a

540 threshold e-value of $\leq 1 \mathrm{e}^{-40}$. In addition, we annotated all candidate sequences identified in $C$.

541 secundus and M. natalensis against the Pfam database (Pfam A, release 30) using hmmscan

542 (HMMER v.3.1b2). 
543 To infer phylogenetic gene trees, we merged for each COG the COGs (amino-acid level) of the

544 seven species listed above with the putatively orthologous amino-acid sequences of $C$. secundus and

545 M. natalensis. We generated multiple sequence alignments for a total of 29 genes of interest

546 applying MAFFT (G-INS-i, see above). Ambiguously aligned sequence sections were identified

547 with Aliscore (v. 2 [94,95]; settings: -r: 10000000 and otherwise defaults) and removed with Alicut

548 (v. 2.3, https://www.zfmk.de/en/research/research-centres-and-groups/utilities; masked alignments

549 provided as Supplementary Archive S1 (deposited at DRYAD, doi available upon acceptance of the

550 manuscript). Phylogenetic trees were inferred with IQ-TREE (1.7-beta12 [96] for each gene. The

551 best model was selected with the implemented ModelFinder [97] from all available nuclear models

552 implemented in IQ-TREE plus the two protein mixture models LG4M and LG4X [98] based on the

553 Bayesian Information Criterion (BIC). We applied default settings for rates and the number of rate

554 categories. Statistical support was inferred from 2,000 non-parametric bootstrap replicates.

555 Unrooted trees with the bootstrap support mapped were visualized with Seaview (v4.5.4 [99]) and

556 provided in Newick Format with Supplementary Archive S1 at DRYAD (doi available upon

557 acceptance of the manuscript).

558

559 Ethics approval and consent to participate

560 Not applicable

561 Consent for publication

562 Not applicable

563 Competing interests

564 The authors declare that they have no competing interests.

565

566 Availability of data and materials 
567 Raw sequence reads are deposited on NCBI (Bioproject XXX, BioSample Accessions see

568 Additional File 2, Table S1 - Bioproject numbers and BioSample Accessions will be provided

569 when obtained from NCBI). Additional supplementary data are deposited on the Dryad digital

570 repository DRYAD (DOI provided upon acceptance of the final manuscript). For Supplementary

571 Material please contact: judith.korb@biologie.uni-freiburg.de

572

\section{Funding}

574 This research was supported by the Deutsche Forschungsgemeinschaft by grants to JK (DFG;

575 KO1895/16-1; KO1895/20-1), one within the Research Unit FOR2281.

576

\section{Contributions}

578 JK designed the study, JK and MMK collected and identified the termite samples, MMK performed

579 all transcriptome analyses, KM helped with gene identification, with data processing and inferred

580 gene trees, JK did survival analysis and supervised the study, all authors wrote the paper.

\section{Acknowledgments}

583 We thank Florentine Schaub for assistance in the field and wet lab, Daniela Schnaiter for termite

584 colony maintenance, Charles Darwin University (Australia), and especially S. Garnett and the

585 Horticulture and Aquaculture team, provided logistic support to collect C. secundus. The Parks and

586 Wildlife Commission, Northern Territory, the Department of the Environment, Water, Heritage and

587 the Arts gave permission to collect. Permit numbers 2002 until 2016, export permit numbers:

588 PWS2001_1508, PWS2003_39852, PWS2004_5769, PWS2007_4154, PWS2010_6997,

589 PWS2014_001342, PWS2016_001559; collection permit numbers: 15656, 18310, 26851, 30073, 
$59036401,51402,59044$. The study was conducted according to the Nagoya protocol. KM thanks

591 Ondrej Hlinka and the CSIRO IM\&T HPC Cluster team.

592

\section{References}

594 1. Jones OR, Scheuerlein A, Salguero-Gómez R, Camarda CG, Schaible R, Casper BB et al.

595 Diversity of ageing across the tree of life. Nature. 2014; 505: 169-173.

596 doi:10.1038/nature12789.

597 2. Linford NJ, Bilgir C, Ro J, Pletcher SD. Measurement of lifespan in Drosophila

598 melanogaster. J Vis Exp. 2013;71. doi:10.3791/50068.

599 3. Ungvari Z, Ridgway I, Philipp EER, Campbell CM, McQuary P, Chow T, et al. Extreme

600 longevity is associated with increased resistance to oxidative stress in Arctica islandica, the

601 longest-living non-colonial animal. J Gerontol A Biol Sci Med Sci. 2011;66:741-50. doi:

$602 \quad 10.1093 /$ gerona/glr044.

603 4. Rose MR. Evolutionary biology of aging. Oxford University Press; 1991; ill.; 25 cm.

604 5. Keller L, Genoud M. Extraordinary lifespans in ants: a test of evolutionary theories of 605 ageing. Nature. 1997;389:958-60. doi: 10.1038/40130.

606 6. Keller L. Queen lifespan and colony characteristics in ants and termites. Insectes Soc.

607 1998;45:235-46.. doi: 10.1007/s000400050084.

608 7. Toth AL, Sumner S, Jeanne RL. Patterns of longevity across a sociality gradient in vespid 609 wasps. Curr Opin insect Sci. 2016;16:28-35. doi: 10.1016/j.cois.2016.05.006. 
610 8. Korb J, Thorne B. Sociality in Termites. In: Rubenstein DR, Abbot P, editors. Comparative

611 Social Evolution. Cambridge: Cambridge University Press; 2017. p. 124-53. doi:

$612 \quad 10.1017 / 9781107338319.006$.

613 9. Corona M, Velarde RA, Remolina S, Moran-Lauter A, Wang Y, Hughes KA, et al.

614 Vitellogenin, juvenile hormone, insulin signaling, and queen honey bee longevity. Proc Natl

$615 \quad$ Acad Sci U S A. 2007;104:7128-33. doi: 10.1073/pnas.0701909104.

616 10. Rascon B, Mutti NS, Tolfsen C, Amdam G V. Honey bee life history plasticity:

617 Development, behavior, and aging. In: Flatt T, Heyland A, editors. Mechanisms of Life

618 History Evolution: The Genetics and Physiology of Life History Traits and Trade-Offs. New

619 York: Oxford Univ Press; 2011.p. 253-66.

620 11. Rodrigues MA, Flatt T. Endocrine uncoupling of the trade-off between reproduction and

621 somatic maintenance in eusocial insects. Curr Opin Insect Sci. 2016;16:1-8. doi: 10.

622 12. Weitekamp CA, Libbrecht R, Keller L. Genetics and evolution of social behavior in insects.

623 Annu. Rev. Genet. 2017;51:219-39. doi: 10.1146/annurev-genet-120116-024515.

624 13. Maekawa K, Ishitani K, Gotoh H, CornetteR, Miura T. Juvenile Hormone titre and

625 vitellogenin gene expression related to ovarian development in primary reproductives

626 compared with nymphs and nymphoid reproductives of the termite Reticulitermes speratus.

627 Physiol Entomol. 2010;35:52-8. doi:10.1111/j.1365-3032.2009.00711.x.

628 14. Korb J. Juvenile Hormone: A Central Regulator of Termite Caste Polyphenism. In: Zayed

629 A, Kent CFBT-A in IP, editors. Genomics, Physiology and Behaviour of Social Insects.

630 Academic Press; 2015. p. 131-61. doi: 10.1016/bs.aiip.2014.12.004. 
631 15. Tasaki E, Mitaka Y, Nozaki T, Kobayashi K, Matsuura K, Iuchi Y. High expression of the 632 breast cancer susceptibility gene BRCA1 in long-lived termite kings. Aging (Albany NY). 633 2018;10:2668-83. doi: 10.18632/aging.101578.

634 16. Wu J, Lu L-Y, Yu X. The role of BRCA1 in DNA damage response. Protein Cell. 635 2010;1:117-23. doi: 10.1007/s13238-010-0010-5.

636 17. Tasaki E, Kobayashi K, Matsuura K, Iuchi Y. An Efficient Antioxidant System in a Long637 Lived Termite Queen. PLoS One. 2017;12:e0167412. doi: 10.1371/journal.pone.0167412.

638 18. Tasaki E, Kobayashi K, Matsuura K, Iuchi Y. Long-Lived Termite Queens Exhibit High 639 Cu/Zn-Superoxide Dismutase Activity. Oxid Med Cell Longev. 2018;2018:5127251. doi: $640 \quad 10.1155 / 2018 / 5127251$.

641 19. Münch D, Amdam G V, Wolschin F. Ageing in a eusocial insect: molecular and 642 physiological characteristics of life span plasticity in the honey bee. Funct Ecol. $643 \quad$ 2008;22:407-21. doi:10.1111/j.1365-2435.2008.01419.x.

644 20. Lucas ER, Keller L. Ageing and somatic maintenance in social insects. Curr Opin Insect Sci. 645 2014;5:31-6. doi: 10.1016/j.cois.2014.09.009.

646 21. de Verges J, Nehring V. A critical look at proximate causes of social insect senescence: 647 damage accumulation or hyperfunction? Curr Opin Insect Sci. 2016;16:69-75. doi:

$648 \quad$ 10.1016/j.cois.2016.05.003.

649 22. Elsner D, Meusemann K., Korb J.Longevity and transposon defense, the case of termite 650 reproductives. Proc Natl Acad Sci U S A. 2018;115:5504-9. doi: 10.1073/pnas.1804046115 
651

652

654

655

656

657

658

660

661

662

663

664

665

666

667

668

669

670

671

672

673

23. Monroy Kuhn JM, Meusemann K, Korb J. Long live the queen, the king and the commoner? Transcript expression differences between old and young in the termite Cryptotermes secundus. PLoS One. 2019;14:e0210371. doi: 10.1371/journal.pone.0210371.

24. Evans DS, Kapahi P, Hsueh W-C, Kockel L. TOR signaling never gets old: aging, longevity and TORC1 activity. Ageing Res Rev. 2011;10:225-37. doi: 10.1016/j.arr.2010.04.001.

25. Partridge L, Alic N, Bjedov I, Piper MD. Ageing in Drosophila: the role of the insulin/Igf and TOR signalling network. Exp Gerontol. 2011;46:376-81. doi:

$$
\text { 10.1016/j.exger.2010.09.003. }
$$

26. Antikainen H, Driscoll M, Haspel G, Dobrowolski R. TOR-mediated regulation of metabolism in aging. Aging Cell. 2017;16:1219-33. doi: 10.1111/acel.12689.

27. Libbrecht R, Corona M, Wende F, Azevedo DO, Serrão, JR, Keller L. Interplay between insulin signaling, juvenile hormone, and vitellogenin regulates maternal effects on polyphenism in ants. Proc Natl Acad Sci U S A. 2013;110:11050-55. doi: 10.1073/pnas.1221781110.

28. Mutti NS, Dolezal AG, Wolschin F, Mutti JS, Gill KS, Amdam GV. IRS and TOR nutrientsignaling pathways act via uvenile hormone to influence honey bee caste fate. J Experim. Biol. 2011;214:3977-84. doi: 10.1242/jeb.061499.

29. Amdam GV, Norberg K, Fondrk MK, Page RE Jr. Reproductive ground plan may mediate colony-level selection effects on individual foraging behavior in honey bees. Proc Natl Acad Sci U S A. 2004;101:11350-55. doi: 10.1073/pnas.0403073101.

30. Warner MR, Qiu L, Holmes MJ, Mikheyev AS, Linksvayer TA. Convergent eusocial evolution is based on a shared reproductive groundplan plus lineage-specific plastic genes. Nat. Commun. 2019;10:2651. doi: 10.1038/s41467-019-10546-w. 
674 31. Chandra V, Fetter-Pruneda I, Oxley PR, Ritger A, McKenzie S, Libbrecht R, Kronauer

675 DJC.Social regulation of insulin signaling and the evolution of eusociality in ants. Science

$676 \quad 2018 ; 361: 398-402$. doi: 10.1126/science.aar5723.

677 32. Kim K, Lee YS, Carthew RW. Conversion of pre-RISC to holo-RISC by Ago2 during 678 assembly of RNAi complexes. RNA. 2007;13:22-9. doi: 10.1261/rna.283207.

679 33. Sabin LR, Zhou R, Gruber JJ, Lukinova N, Bambina S, Berman A, et al. Ars2 regulates both 680 miRNA- and siRNA- dependent silencing and suppresses RNA virus infection in Drosophila. Cell. 2009;138:340-51. doi: 10.1016/j.cell.2009.04.045.

34. Ivanov DK, Escott-Price V, Ziehm M, Magwire MM, Mackay TFC, Partridge L, et al. Longevity GWAS Using the Drosophila Genetic Reference Panel. J Gerontol A Biol Sci Med Sci. 2015;70:1470-8. doi: 10.1093/gerona/glv047. Molecular Approach. 2nd edition. Sunderland, MA: Sinauer Associates; 2000. 2010;2:299-314. doi:10.1007/s13148-010-0017-z.

694 39. Steunou A-L, Rossetto D, Côté J. Regulating Chromatin by Histone Acetylation BT - 

splicing is essential for the unfolded protein response (UPR) in Drosophila melanogaster. Neurodegenerative Disorders and Its Potential Therapeutic Significance. Front Mol Neurosci. 2017;10:187. doi:10.3389/fnmol.2017.00187. with Nrf2 to regulate antioxidant signaling and cell survival. J Exp Med. 2013;210:1529-44.

43. Piatek MJ, Werner A. Endogenous siRNAs: regulators of internal affairs. Biochem Soc Trans. 2014;42:1174-9. doi:10.1042/BST20140068. lysine 16 acetylation regulates cellular lifespan. Nature. 2009;459:802-7. doi:

711 45. Wood JG, Hillenmeyer S, Lawrence C, Chang C, Hosier S, Lightfoot W, et al. Chromatin 712 remodeling in the aging genome of Drosophila. Aging Cell. 2010;9:971-8.. doi:

$713 \quad$ 10.1111/j.1474-9726.2010.00624.x.

714 46. Larson K, Yan S-J, Tsurumi A, Liu J, Zhou J, Gaur K, et al. Heterochromatin formation 715 promotes longevity and represses ribosomal RNA synthesis. PLoS Genet. 2012;8:e1002473. 716 doi: 10.1371/journal.pgen.1002473.

717 47. Wood JG, Helfand SL. Chromatin structure and transposable elements in organismal aging. 718 Front Genet. 2013;4:274. doi: 10.3389/fgene.2013.00274. 
719 48. Chen H, Zheng X, Xiao D, Zheng Y. Age $\square$ associated de $\square$ repression of retrotransposons in 720 the Drosophila fat body, its potential cause and consequence. Aging Cell. 2016;15:542-52. 721 doi:10.1111/acel.12465.

722 49. Wood JG, Jones BC, Jiang N, Chang C, Hosier S, Wickremesinghe P, et al. Chromatin723 modifying genetic interventions suppress age-associated transposable element activation and 724 extend life span in Drosophila. Proc Natl Acad Sci U S A. 2016;113:11277-82 doi:

$725 \quad$ 10.1073/pnas.1604621113.

726 50. Liu Y, Ye X, Jiang F, Liang C, Chen D, Peng J, et al. C3PO, an endoribonuclease that 727 promotes RNAi by facilitating RISC activation. Science. 2009;325:750-3. doi:

$728 \quad 10.1126 /$ science. 1176325 .

729 51. Kandasamy SK, Zhu L, Fukunaga R. The C-terminal dsRNA-binding domain of Drosophila 730 Dicer-2 is crucial for efficient and high-fidelity production of siRNA and loading of siRNA 731 to Argonaute2. RNA. 2017;23:1139-53. doi: 10.1261/rna.059915.116.

732 52. Tsuboyama K, Tadakuma H, Tomari Y. Conformational activation of Argonaute by distinct 733 yet coordinated actions of the Hsp70 and Hsp90 chaperone systems. Mol Cell. 2018;70:722734 729.e4. doi: 10.1016/j.molcel.2018.04.010.

735 53. Liu Y, Beyer A, Aebersold R. On the dependency of cellular protein levels on mRNA 736 abundance. Cell. 2016;165:535-50. doi: 10.1016/j.cell.2016.03.014.

737 54. Cao JQ, Tong WS, Yu HY, Tobe SS, Bendena WG, Hui JHL. Chapter Three - The Role of 738 MicroRNAs in Drosophila Regulation of Insulin-Like Peptides and Ecdysteroid Signalling:

739 Where Are We Now? In: Verlinden HBT-A in IP, editor. Insect Epigenetics. Academic $740 \quad$ Press; 2017. p. 55-85. doi: 10.1016/bs.aiip.2017.02.002. 
741 55. Laplante M, Sabatini DM. mTOR signaling at a glance. J Cell Sci. 2009;122 Pt 20:3589-94.

742 doi: $10.1242 /$ jcs.051011.

743 56. Zhao J, Zhai B, Gygi SP, Goldberg AL. mTOR inhibition activates overall protein

744 degradation by the ubiquitin proteasome system as well as by autophagy. Proc Natl Acad

$745 \quad$ Sci U S A. 2015;112:15790-7. doi: 10.1073/pnas.1521919112.

746 57. Ichimura Y, Waguri S, Sou Y-S, Kageyama S, Hasegawa J, Ishimura R, et al.

747 Phosphorylation of p62 activates the Keap1-Nrf2 pathway during selective autophagy. Mol

$748 \quad$ Cell. 2013;51:618-31. doi: 10.1016/j.molcel.2013.08.003.

749 58. Aramburu J, Ortells MC, Tejedor S, Buxade M, Lopez-Rodriguez C. Transcriptional

750 regulation of the stress response by mTOR. Sci Signal. 2014;7:re2. doi:

$751 \quad 10.1126 /$ scisignal.2005326.

752 59. Tsakiri EN, Sykiotis GP, Papassideri IS, Terpos E, Dimopoulos MA, Gorgoulis VG, et al.

753 Proteasome dysfunction in Drosophila signals to an Nrf2-dependent regulatory circuit

$754 \quad$ aiming to restore proteostasis and prevent premature aging. Aging Cell. 2013;12:802-13.

755 doi: 10.1111/acel.12111.

756 60. Sykiotis GP, Bohmann D. Keap1/Nrf2 signaling regulates oxidative stress tolerance and

757 lifespan in Drosophila. Dev Cell. 2008;14:76-85. doi: 10.1016/j.devcel.2007.12.002.

758 61. Tullet JMA, Hertweck M, An JH, Baker J, Hwang JY, Liu S, et al. Direct inhibition of the

759 longevity-promoting factor SKN-1 by insulin-like signaling in C. elegans. Cell.

$760 \quad$ 2008;132:1025-38. doi: 10.1016/j.cell.2008.01.030.

761 62. Tolfsen CC, Baker N, Kreibisch C, Amdam GV. Flight restriction prevents associative 762 learning deficits but no changes in brain protein-adduct formation during honeybee ageing .

$763 \quad$ J Exp Biol. 2011;214:1322-32. 
764 63. Parker JD, Parker KM, Sohal BH, Sohal RS, Keller L. Decreased expression of Cu-Zn

765 superoxide dismutase 1 in ants with extreme lifespan. Proc Natl Acad Sci U S A.

$766 \quad$ 2004;101:3486-9. doi:10.1073/pnas.0400222101.

767 64. Heinze J, Schrempf A. Terminal investment: individual reproduction of ant queens increases

768 with age. PLoS One. 2012;7:e35201. doi: 10.1371/journal.pone.0035201.

769 65. Korb J, Lenz M. Reproductive decision-making in the termite, Cryptotermes secundus

770 (Kalotermitidae), under variable food conditions. Behav Ecol. 2004;15:390-5. doi:

$771 \quad$ 10.1093/beheco/arh033.

772 66. Korb J, Katrantzis S. Influence of environmental conditions on the expression of the sexual

773 dispersal phenotype in a lower termite: implications for the evolution of workers in termites.

774 Evol Dev. 2004;6:342-52. doi: 10.1111/j.1525-142X.2004.04042.x.

775 67. IBM Corp. Released 2015. IBM SPSS Statistics for Windows, Version 23.0. Armonk, NY:

776 IBM Corp.

777 68. Andrews S. FastQC: a quality control tool for high throughput sequence data. 2010.

778 http://www.bioinformatics.babraham.ac.uk/projects/fastqc.

779 69. Bray NL, Pimentel H, Melsted P, Pachter L. Near-optimal probabilistic RNA-seq

780 quantification. Nat Biotech. 2016;34:525-7. doi:10.1038/nbt.3519.

781 70. Harrison MC, Jongepier E, Robertson HM, Arning N, Bitard-Feildel T, Chao H, et al.

782 Hemimetabolous genomes reveal molecular basis of termite eusociality. Nat Ecol Evol.

783 2018;2:557-66. doi: 10.1038/s41559-017-0459-1.

784 71. Love MI, Huber W, Anders S. Moderated estimation of fold change and dispersion for 785 RNA-seq data with DESeq2. Genome Biol. 2014;15:550. doi: 10.1186/s13059-014-0550-8. 
786 72. R Core Team. 2018 R: A Language and Environment for Statistical Computing (R

787 Foundation for Statistical Computing, Vienna). https://www.R-project.org/.

788 73. Nueda MJ, Martorell-Marugan J, Marti C, Tarazona S, Conesa A. Identification and

789 visualization of differential isoform expression in RNA-seq time series. Bioinformatics.

2018;34:524-6. doi: 10.1093/bioinformatics/btx578.

791 74. Nueda MJ, Tarazona S, Conesa A. Next maSigPro: updating maSigPro bioconductor

792 package for RNA-seq time series. Bioinformatics. 2014;30:2598-602.

793 doi:10.1093/bioinformatics/btu333.

794 75. Langfelder P, Horvath S. WGCNA: an R package for weighted correlation network analysis.

795 BMC Bioinformatics. 2008;9:559. doi:10.1186/1471-2105-9-559.

796 76. Zhang B, Horvath S. A general framework for weighted gene co-expression network

797 analysis. Stat Appl Genet Mol Biol. 2005;4:Article17. doi: 10.2202/1544-6115.1128.

798 77. Horvath S, Dong J. Geometric interpretation of gene coexpression network analysis. PLoS

799 Comput Biol. 2008;4:e1000117. doi: 10.1371/journal.pcbi.1000117.

800 78. Yip AM, Horvath S. Gene network interconnectedness and the generalized topological

801 overlap measure. BMC Bioinformatics. 2007;8:22. doi:10.1186/1471-2105-8-22.

802 79. Langfelder P, Zhang B, Horvath S. Defining clusters from a hierarchical cluster tree: the

803 Dynamic Tree Cut package for R. Bioinformatics. 2008;24:719-20. doi:

$804 \quad 10.1093 /$ bioinformatics/btm563.

805 80. Shannon P, Markiel A, Ozier O, Baliga NS, Wang JT, Ramage D, et al. Cytoscape: a

806 software environment for integrated models of biomolecular interaction networks. Genome

807 Res. 2003;13:2498-504. doi: 10.1101/gr.1239303. 
808 81. Kucera M, Isserlin R, Arkhangorodsky A, Bader GD. AutoAnnotate: A Cytoscape app for

809 summarizing networks with semantic annotations. F1000Research. 2016;5:1717.

$810 \quad$ doi:10.12688/f1000research.9090.1.

811 82. Enright AJ, Van Dongen S, Ouzounis CA. An efficient algorithm for large-scale detection of 812 protein families. Nucleic Acids Res. 2002;30:1575-84.

813 83. Maere S, Heymans K, Kuiper M. BiNGO: a Cytoscape plugin to assess overrepresentation 814 of Gene Ontology categories in Biological Networks. Bioinformatics. 2005;21:3448-9. doi:

$815 \quad$ 10.1093/bioinformatics/bti551.

816 84. Benjamini Y, Hochberg Y. Controlling the False Discovery Rate: A Practical and Powerful 817 Approach to Multiple Testing. J R Stat Soc Ser B. 1995;57:289-300.

818 http://www.jstor.org/stable/2346101.

819 85. Finn RD, Coggill P, Eberhardt RY, Eddy SR, Mistry J, Mitchell AL, et al. The Pfam protein 820 families database: towards a more sustainable future. Nucleic Acids Res. 2016;44:D279-85. 821 doi: 10.1093/nar/gkv1344.

822 86. Eddy SR. Accelerated Profile HMM Searches. PLoS Comput Biol. 2011;7:e1002195. doi: $823 \quad$ 10.1371/journal.pcbi.1002195.

824 87. Jones P, Binns D, Chang H-Y, Fraser M, Li W, McAnulla C, et al. InterProScan 5: genome825 scale protein function classification. Bioinformatics. 2014;30:1236-40. doi:

$826 \quad$ 10.1093/bioinformatics/btu031.

827 88. Altschul SF, Gish W, Miller W, Myers EW, Lipman DJ. Basic local alignment search tool. J 828 Mol Biol. 1990;215:403-10. doi: 10.1016/S0022-2836(05)80360-2. 
829 89. Terrapon N, Li C, Robertson HM, Ji L, Meng X, Booth W, et al. Molecular traces of

830 alternative social organization in a termite genome. Nat Commun. 2014;5:3636. doi:

$831 \quad 10.1038 /$ ncomms4636.

832 90. Hubley R, Finn RD, Clements J, Eddy SR, Jones TA, Bao W, et al. The Dfam database of 833 repetitive DNA families. Nucleic Acids Res. 2016;44:D81-9. doi: 10.1093/nar/gkv1272.

834 91. Wheeler TJ, Eddy SR. nhmmer: DNA homology search with profile HMMs.

835 Bioinformatics. 2013;29:2487-9. doi: 10.1093/bioinformatics/btt403.

836 92. Zdobnov EM, Tegenfeldt F, Kuznetsov D, Waterhouse RM, Simao FA, Ioannidis P, et al.

837 OrthoDB v9.1: cataloging evolutionary and functional annotations for animal, fungal, plant,

838 archaeal, bacterial and viral orthologs. Nucleic Acids Res. 2017;45:D744-9. doi:

$839 \quad 10.1093 /$ nar/gkw1119.

840 93. Katoh K, Standley DM. MAFFT multiple sequence alignment software version 7:

841 improvements in performance and usability. Mol Biol Evol. 2013;30:772-80.

842 doi:10.1093/molbev/mst010-

843 94. Misof B, Misof K. A Monte Carlo approach successfully identifies randomness in multiple

844 sequence alignments: a more objective means of data exclusion. Syst Biol. 2009;58:21-34.

845 doi: 10.1093/sysbio/syp006.

846 95. Kück P, Meusemann K, Dambach J, Thormann B, von Reumont BM, Wägele JW et al.

847 Parametric and non-parametric masking of randomness in sequence alignments can be

848 improved and leads to better resolved trees. Front Zool. 2010;7:10. doi: 10.1186/1742-9994-

$849 \quad 7-10$.

850 96. Nguyen L-T, Schmidt HA, Von Haeseler A, Minh BQ. IQ-TREE: a fast and effective 851 stochastic algorithm for estimating maximum-likelihood phylogenies. Mol Biol Evol. 
2015;32:268-74. doi: 10.1093/molbev/msu300.

853 97. Kalyaanamoorthy S, Minh BQ, Wong TKF, von Haeseler A, Jermiin LS. ModelFinder: fast

854 model selection for accurate phylogenetic estimates. Nat Methods. 2017;14:587-9. doi:

$855 \quad 10.1038 /$ nmeth.4285.

856 98. Le SQ, Dang CC, Gascuel O. Modeling protein evolution with several amino acid

857 replacement matrices depending on site rates. Mol Biol Evol. 2012;29:2921-36. doi:

$858 \quad 10.1093 / \mathrm{molbev} / \mathrm{mss} 112$

859 99. Gouy M, Guindon S, Gascuel O. SeaView version 4: A multiplatform graphical user

860 interface for sequence alignment and phylogenetic tree building. Mol Biol Evol.

2010;27:221-4. doi: 10.1093/molbev/msp259. 


\section{FIGURE LEGENDS}

\section{Figure 1. Survival plot of $C$. secundus queens.}

864 Shown is the age-dependent survival probability of queens. The median longevity of queens in the

865 laboratory after successful colony establishment was estimated with Kaplan Meier survival analysis

866 to be 12 years (mean longevity: 11.1 years). The maximum lifespan was 13 years. After an age of

867 around 11 years, life expectancy declines rapidly; out of eight queens with a potential age $\geq 12$

868 years, all had died, except one 13-year old queen.

869

870 Figure 2. Median expression profiles of DETs assigned to Iso-MaSigPro clusters.

871 Iso-MaSigPro grouped the differentially expressed transcripts (DETs) into six clusters. DETs of

872 cluster 1, 4 and 6 were especially highly expressed in old queens, while those of cluster 3 and 5

873 characterized middle-aged queens and those of cluster 2 young queens. The expression values

874 correspond to normalized counts (see Methods). The youngest queen (age: 2 years) was taken as

875 time step zero and each of the subsequent older queens (based on chronological age) were

876 considered to be one time step older. One age class (time step 8; age: 10 years) consisted of two

877 samples.

878

879 Figure 3. Young subnetwork highlighting Iso-MaSigPro DETs.

880 Shown is WGCNA-based co-expression network of transcripts, which contains DETs characterising

881 young and middle-aged queens and their one- and two-step neighbors (i.e., young subnetwork; for

882 more information, see text and Additional File 1, Figure S1 and S2). Highlighted are the Iso-

883 MaSigPro DETs of cluster 2, 3, and 5, characterizing young and middle-aged queens (see insert;

884 Fig. 2). Node colors correspond to the WGCNA modules. Transposable element (TE) related

885 transcripts are highlighted with a '?'. Transcripts with an asterisk indicate 1:1 orthologs $(C$. 
886 secundus and D. melanogaster). Connection length and width do not have a meaning. Red circles

887 indicate transcripts discussed in the text.

889 Figure 4. Genes related to protein synthesis that were found in the old subnetwork.

890 Shown are genes that have been related to various processes of protein synthesis, from initiation,

891 and elongation to termination. For all genes listed, corresponding transcripts were present in the old

892 subnetwork of $C$. secundus queens. Figure modified after [35].

893

894 Figure 5. Genes related to the proteasome complex that were found in the old subnetwork.

895 Shown are genes that have been related to the proteasome complex. The textbox in red indicates

896 subunits, for which we found transcripts in the old subnetwork. Figure modified after [36].

897

898 Figure 6. Transcription- and proteostasis-related expression pattern in old Cryptotermes

899 secundus queens.

900 Depicted is the expression patterns of genes related to transcription and proteostasis for old $C$.

901 secundus queens (red arrows) in contrast to that reported for other species (grey arrows). Old $C$.

902 secundus queens were characterized by a very strong proteostasis signal indicative of an

903 upregulation of protein degradation, together with protein synthesis and protein folding. This is

904 unusual because old organisms are typically characterized by a downregulation of these processes.

905 The simultaneous activation of protein synthesis and degradation in old C. secundus queens can be

906 explained by the activity of the transcription factor cnc/Nrf-2 (for more details, see text). The inner

907 cycle arrows depict the protein life cycle; dashed arrows indicate the special case when mistakes/

908 errors occur. After a protein is degraded, its components are recycled.

909 


\section{Figure 7. Aging signal of $\boldsymbol{C}$. secundus queens in relation to known aging pathways.}

911 Shown are simplified IIS (insulin/insulin-like growth factor signaling; blue), TOR (target of

912 rapamycin; green), and ecdysone (brown) pathways and their interactions with an emphasis on $\mathbf{A}$.

913 ecdysone biosynthesis and B. protein synthesis and degradation. Red encircled genes were members

914 of the old subnetwork, and thus highly expressed in old queens. Important genes that regulate

915 protein synthesis and degradation are depicted in white. In short, the TOR pathway controls cell

916 growth and metabolism in response to amino acid availability. It is generally composed of two main

917 complexes: TORC1 and TORC2 [55]. Activation of TORC1 promotes mRNA translation, for

918 instance, via S6K /eIF4B / eIF-4a and 4E-BP / eIF4E. Additionally, active TORC1 inhibits

919 autophagy by targeting upstream components necessary for autophagy activation, like Atg1. TOR

920 interacts with IIS, which also regulates multiple physiological functions, including aging.

921 Generally, an active IIS pathway can activate the TORC1 complex via phosphorylation and

922 inactivation of Tsc 2 by AKT. AKT inhibits the transcription factor foxo via phosphorylation, which

923 results in the inhibition of transcription of many downstream genes, e.g. involved in lifespan

924 extension, stress response and autophagy. Stress induced $C n c$ can activate TORC1 in a positive

925 feedback loop (big dashed arrow). It may be responsible for the simultaneous upregulation of

926 protein synthesis and degradation. For more information, see text. Figures are adapted after [53-55].

927

928 


\section{Additional Files}

\section{Additional File 1 (Additional_File_1.pdf): Supplementary Figures S1-S10.}

931 Figure S1: Schematic workflow. Details are explained in the Methods.

932 Figure S2: WGCNA modules of co-expressed transcripts that negatively correlate with age.

933 Modules marked with an asterisk were found in the young subnetwork. Modules are named after

934 colors by WGCNA.

935 Figure S3: WGCNA modules of co-expressed transcripts that positively correlate with age.

936 Modules marked with an asterisk were found in the old subnetwork. Modules are named after colors

937 by WGCNA.

938 Figure S4: GO enrichment for the WGCNA module 'tan' that positively correlated with age and

939 which had many old-age DETs. Details are shown for BP (Biological Process), which revealed an

940 enrichment of transcripts for ribosomal and tRNA related functions.

941 Figure S5: Young and old transcript subnetworks corresponding to the second level neighborhood

942 of Iso-MaSigPro DETs. Age-related DETs were located in the WGCNA co-expression network and

943 these DETs and their one- and two-step neighbors (i.e., the 'second level neighborhood') were then

944 extracted from the co-expression network to provide the shown networks.

945 Figure S6: BiNGO GO enrichment (Biological Process) for the young subnetwork. No terms were

946 significantly enriched after correcting for multiple testing (FDR).

947 Figure S7: BiNGO GO enrichment (Biological Process) for the old subnetwork. Colored nodes are

948 GO terms that were significantly enriched after correcting for multiple testing (FDR).

949 Figure S8: BiNGO GO enrichment of (Molecular Function; Cellular Component) for the old

950 subnetwork. Colored nodes are GO terms that were significantly enriched after correcting for

951 multiple testing (FDR). 
952 Figure S9: BiNGO GO enrichment (Biological Process; Molecular Function; Cellular Component)

953 for the 'green' WGCNA module, which is part of the old subnetwork. Colored nodes are GO terms

954 that were significantly enriched after correcting for multiple testing (FDR).

955 Figure S10: BiNGO GO enrichment (Biological Process, Molecular Function; Cellular Component)

956 for the 'paleturquoise' WGCNA module, which is part of the old subnetwork. Nodes in color are

957 GO terms significantly enriched after correcting for multiple testing (FDR).

958

959 Additional File 2 (Additional_File_2.xlsx): Supplementary Tables S1-S4.

960 Table S1: Sample information of samples included in this study.

961 Table S2: Differentially expressed transcripts of the Iso-MaSigPro analysis, separately for cluster 1-

9626.

963 Table S3. Transcripts in the young subnetwork (SNW).

964 Transcripts in the young subnetwork (SNW) classified into major categories; TE-related transcripts

965 of the young subnetwork (SNW).

966 Table S4. Transcripts in the old subnetwork (SNW).

967 Transcripts in the old subnetwork (SNW) classified into major categories; TE-related transcripts of 968 the old subnetwork (SNW).

969

970 Additional File 3 (Additional_File_3.pdf)

971 WGCNA module-age associations. Listed are the eigengene values for each module.

972

973 Additional File 4 (Additional_File_4.xlsx): Supplementary Table S5.

974 Go terms for enriched differentially expressed transcripts (DETs) included in BiNGO module

975 green; BiNGO module paleturquoise; BiNGO module tan; BiNGO module Thistle2; BiNGO 
976 module snow; BiNGO module cyan; BiNGO module deeppink1; BiNGO module navajowhite;

977 BiNGO module blue2_NS; BiNGO module cornflowerblue_NS; BiNGO module pink3_NS;

978 BiNGO module steelblue4_NS.

979

\section{Supplementary Archive 1}

981 The Supplementary Archive includes i) masked multiple sequence alignments (MSAs) of 29

982 selected genes (subdirectory "MSAs" used for ML gene tree inference in FASTA format) in IQ-

983 TREE and ii) inferred ML gene trees (subdirectory "ML_gene_trees" in NEWICK format) for each

984 of the 29 genes including non-parametric statistical bootstrap support. These gene trees additionally

985 helped to ensure a proper assignment of transcripts to repective genes. 


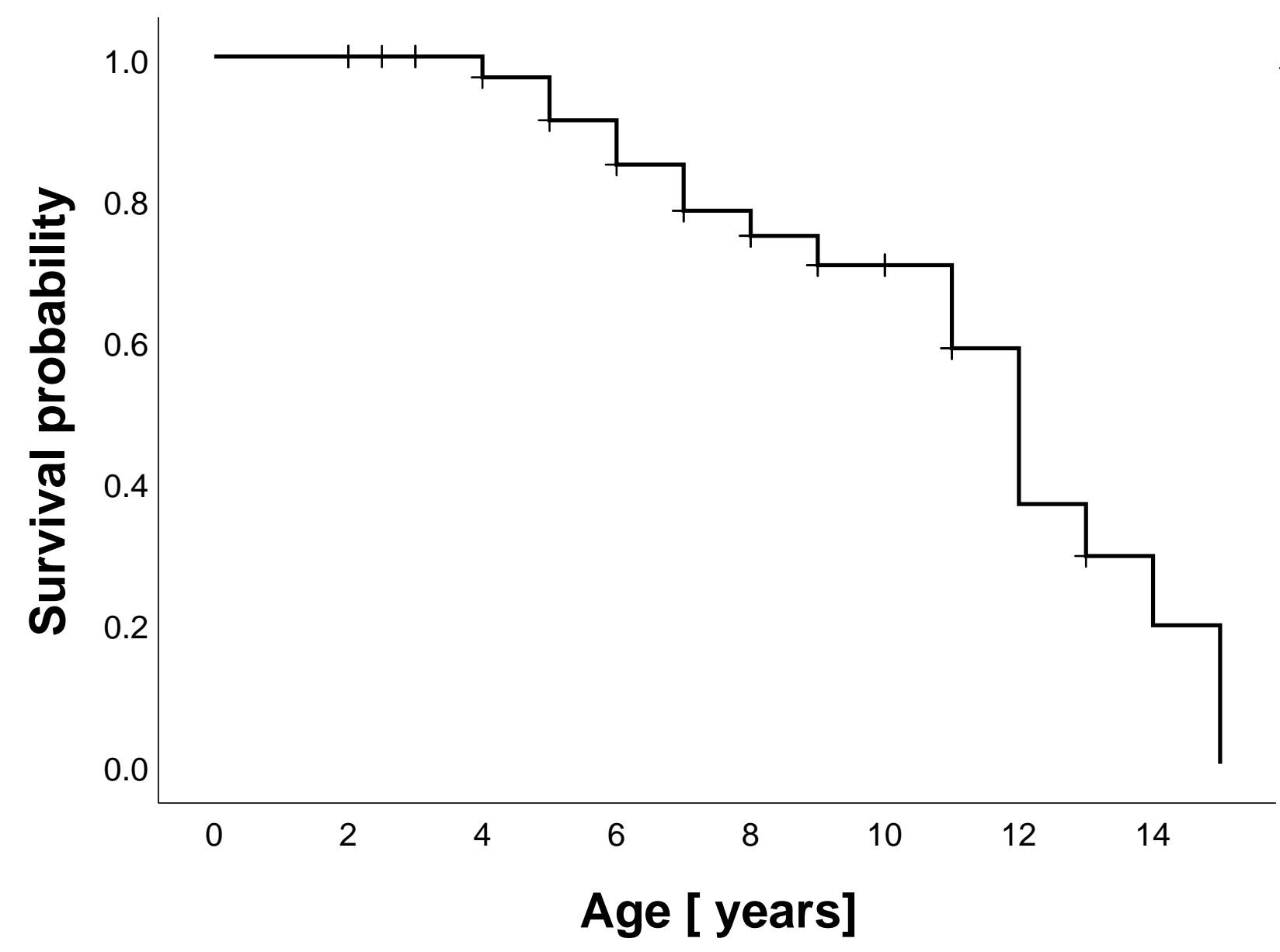

TSurvival Functior + Censored 


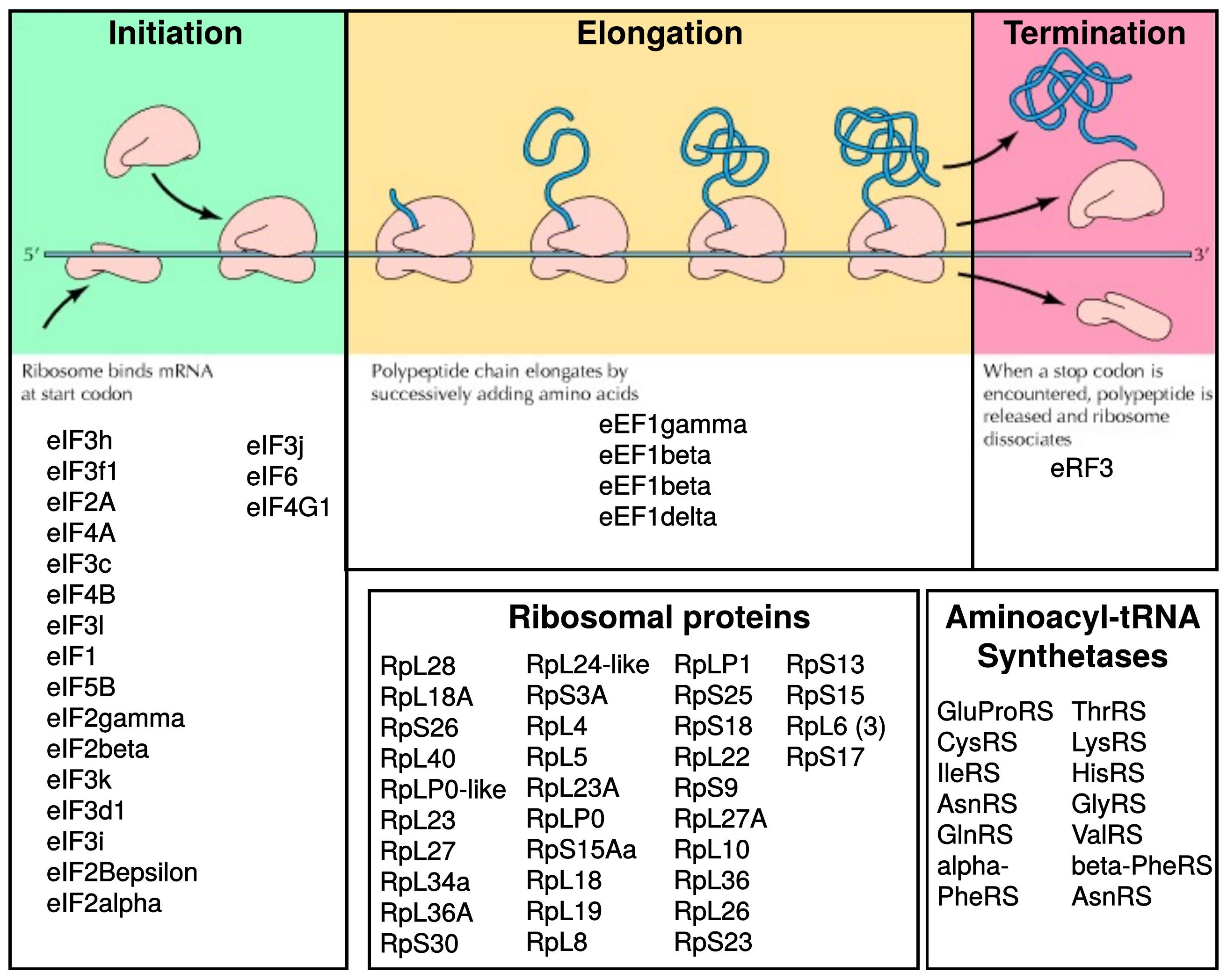




\section{Subunits in the "old" subnetwork}

Rpn3, 5-9, $11,12,15$

니

Rpn10

265

20S core

particle

proteasome

$19 S$

regulatory

particle

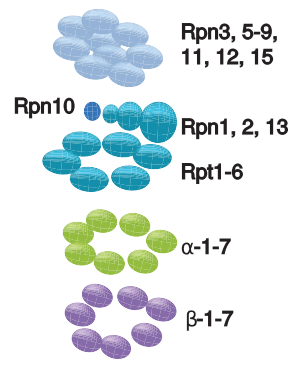

Base

$\alpha$-Ring

$\beta$-Ring
$3,4,5,7$

Rpn3, 7, 8, 9, 11, 12

Rpn2, 10(2)

Rpt1, 2, 3, 4, 5

1-7 
Splicing factors

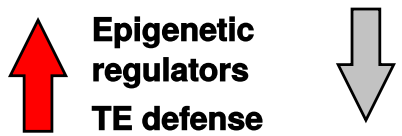

TEs

Transcription

Elongation factors

Termination factors

Aminoacylt-tRNA

synthetases

Ribosomal proteins

Protein Synthesis
Cnc/Nrf-2 xbp1
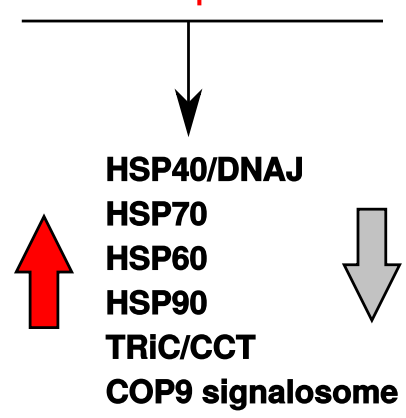

if Upregulated at old age

ป Downregulated at old age

-1 Inhibits

$\rightarrow$ Activates

$\&$ Error

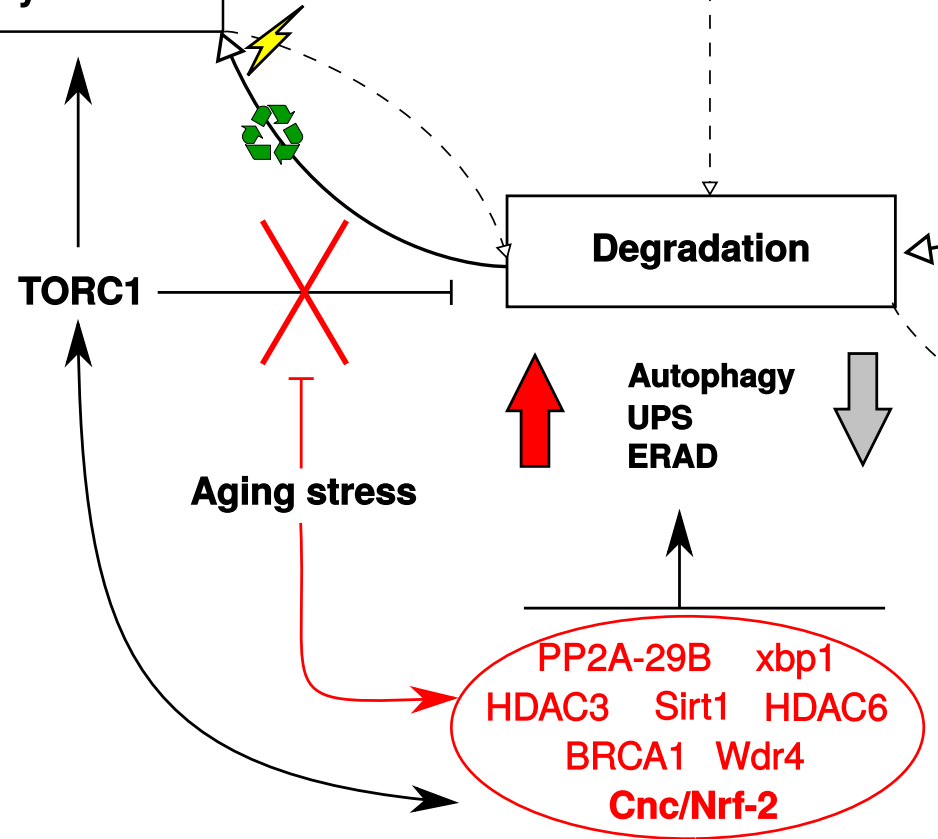



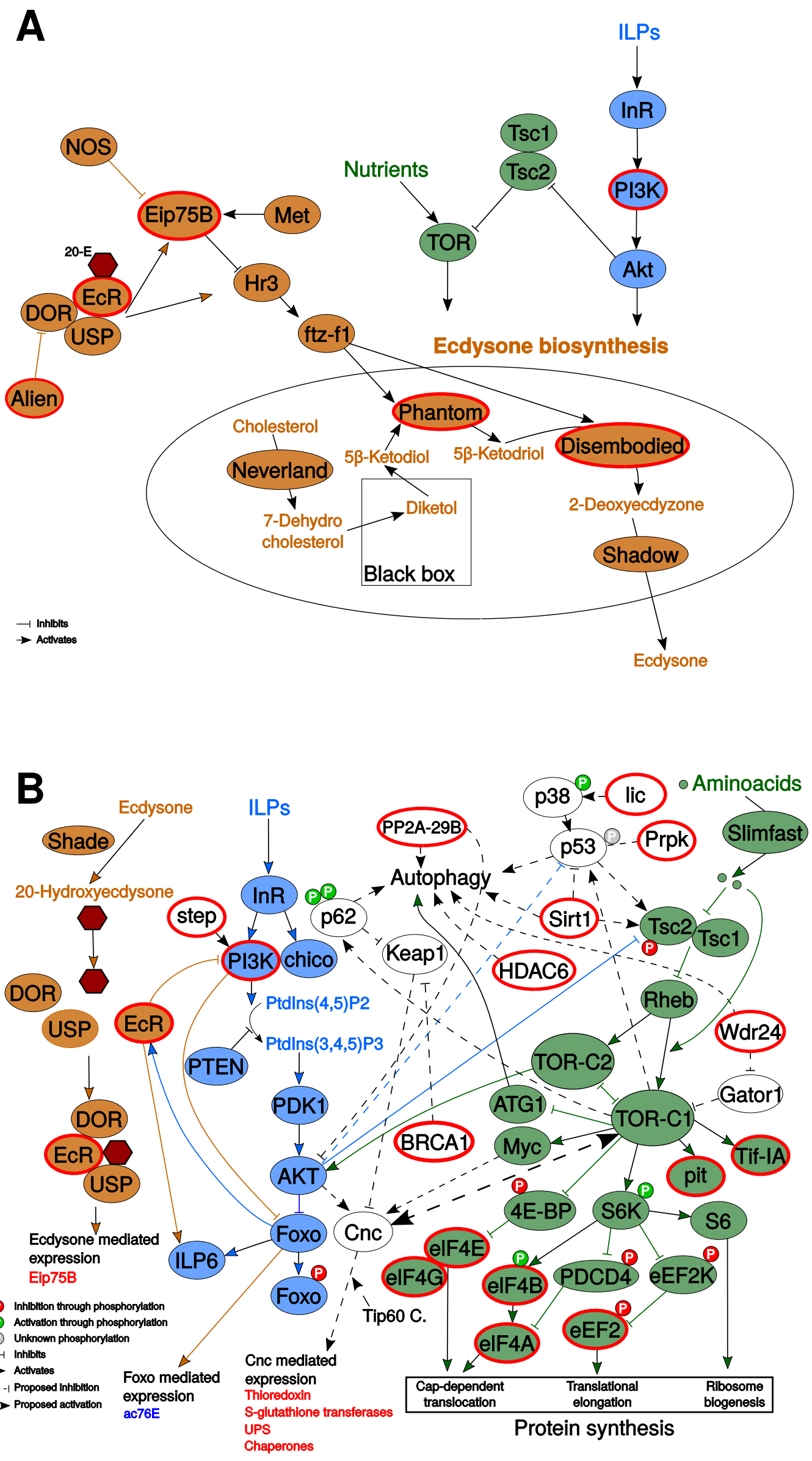